\title{
Sexual behavior among volunteers enrolled in a Phase I HIV vaccine trial: experience of Projet San Francisco in Kigali, Rwanda
}

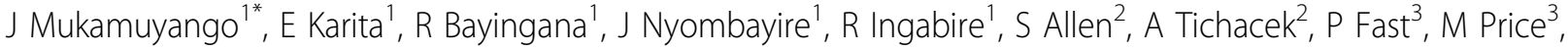 \\ C Schmidt $^{3}$
}

From AIDS Vaccine 2012

Boston, MA, USA. 9-12 September 2012

\section{Background}

Phase 1 HIV vaccine trials are conducted among HIVuninfected, healthy volunteers at low risk for HIV. Study volunteers are counseled to maintain low risk behavior for HIV acquisition. The objective of this study was to assess sexual behavior of volunteers in a Phase 1 vaccine trial, conducted in Kigali, Rwanda.

\section{Methods}

Concordant HIV-negative couples who were counseled and tested together were considered as low risk group for HIV acquisition and were invited to participate in Phase 1 HIV vaccine trial to evaluate safety and immunogenicity of a multiclade HIV-1 DNA plasmid vaccine followed by a recombinant, multiclade HIV-1 adenoviral vector vaccine (IAVI V001). HIV risk reduction counseling, assessment of sexual behavior and screening for sexually transmitted infections (STI) were conducted quarterly. After completion of the vaccine trial, participants and their partners were invited to participate in a long-term 5-year follow-up study from their last vaccination for further assessment of sexual behavior and continuous risk reduction counseling.

\section{Results}

Between November 2005 and May 2006, 57 volunteers (36 men and 21 women) were enrolled in the clinical trial. All volunteers completed their trial visits. During the first twelve months following the first vaccination, none of the study participants reported sex with other partners nor was treated for STI. After unblinding,
55 volunteers agreed to continue in long-term follow up study. Through January 20126 men and 2 women reported having sex at least once with other partners; two were treated for STI, and one subsequently acquired HIV. (HIV incidence rate: 0.3/100 person-years).

\section{Conclusion}

This trial and subsequent follow-up study confirm our previous findings that concordant HIV-negative couples are at low risk of HIV infection and suitable for enrolling in Phase $1 \mathrm{HIV}$ prevention trials. Ongoing HIV risk reduction counseling should be provided during the trial as well as after the trail, if possible.

\section{Author details \\ ${ }^{1}$ Projet San Francisco, Kigali, Rwanda. Emory University, Atlanta, GA, USA. \\ ${ }^{3}$ International AIDS Vaccine Initiative, New York, NY, USA.}

Published: 13 September 2012

doi:10.1186/1742-4690-9-S2-P116

Cite this article as: Mukamuyango et al: Sexual behavior among volunteers enrolled in a Phase I HIV vaccine trial: experience of Projet San Francisco in Kigali, Rwanda. Retrovirology 2012 9(Suppl 2):P116.

${ }^{1}$ Projet San Francisco, Kigali, Rwanda

Full list of author information is available at the end of the article 